\title{
On periodic Jacobi-Perron algorithm over the field of formal power series
}

\author{
Amara Chandoul \\ University of Brasilia, Campus Darcy Ribeiro, Departamento de Matematica, Brasilia DF, Brazil \\ Received: 25 March 2017, Accepted: 11 December 2017 \\ Published online: 28 February 2018.
}

\begin{abstract}
This paper studies the Jacobi-Perron algorithm over the formal power series field $F_{q}\left(\left(X^{-1}\right)\right)$, following E. Dubois. Let $F_{q}[X][\rho]$ be any formal power series field extension with degree $(n+1)$, where $n \in \mathbb{N}$. This should be concerned with the periodicity of the vector $\left(\omega_{1}, \omega_{2}, \ldots, \omega_{n}\right) \in\left(F_{q}[X][\rho]\right)^{n}$ under the iteration of the Jacobi-Perron algorithm. We proved that there is a vector $\left(\omega_{1}, \omega_{2}, \ldots, \omega_{n}\right) \in\left(F_{q}[X][\rho]\right)^{n}$, which is periodic by the Dubois version of the Jacobi-Perron algorithm.

We also proved that there is no algebraic formal series $\omega$ such that the vector $\left(\omega, \omega^{2}\right)$ is 2-periodic by Jacobi-Perron algorithm. Besides, we gave a characterization of the vector $\left(\omega, \omega^{2}\right)$ which is 1 and 3-purely periodic. These results are the analogous of those in the real case. We also conjectured that there is no vector $\left(\omega, \omega^{2}\right)$ which is $2 n$-purely periodic by Jacobi-Perron algorithm and the odd-purely periodic is the same with the cases of 1 and 3-purely periodic.
\end{abstract}

Keywords: Jacobi-Perron Algorithm, Dubois version, Pisot element, algebraic extension, periodic.

\section{Introduction}

In 1907, since Perron introduced the Jacobi-Perron algorithm, which is the simple generalization of continued fractions to finite sets of real numbers, the following conjecture of characterizing the periodicity is still open.

Conjecture 1. Let $\mathbb{K}$ be a real number field of degree $n+1$. Let $\left(\alpha_{1}, \ldots, \alpha_{n}\right) \in \mathbb{K}$ be such that $1, \alpha_{1}, \ldots, \alpha_{n}$ are linearly independent over $\mathbb{Q}$. Then the $n$-tuple $\left(\alpha_{1}, \ldots, \alpha_{n}\right)$ is, eventually becomes periodic by the Jacobi-Perron algorithm.

The case $n=2$ is a special one, since the characteristic equation is irreducible ( see $[14,15]$ ).

Due to Dubois and Paysant-Le Roux [2] it is proved that for any cubic extension of the field $\mathbb{Q}$, there is a pair of numbers $\alpha_{1}, \alpha_{2}$ such that their expansion by Jacobi-Perron algorithm is periodic.

Bouhamza, in $[7,8]$ was able to prove that there is no natural number $m>0$ such that the Jacobi-Perron algorithm of $\left(\sqrt[3]{m}, \sqrt[3]{m}^{2}\right)$ is purely periodical. He proved also that for any extension of the field $\mathbb{Q}$, of degree 4 there is a triple of numbers $\alpha_{1}, \alpha_{2}, \alpha_{3}$ such that their expansion by Jacobi-Perron algorithm is periodic.

Again, R. Paysant-Le Roux and Dubois [3] proved that for any real number field there exists a basis for which we have periodicity. This result depends on properties of the Pisot number.

In the formal power case, Dubois [4] gave a New version of Jacobi-Perron algorithm. He proved that the simultaneous Diophantine properties of the algorithm are stronger than in the real case. But He did not characterize the system of formal power series whose expansion by Jacobi-Perron algorithm is periodic. Furthermore he characterized the vector 
$\left(\omega_{1}, \ldots, \omega_{n}\right)$ which is 1-periodic by his version of Jacobi-Perron algorithm.

In this paper we are able to prove that over any formal power series field extension of degree $(n+1), \mathbf{F}_{q}[X][\rho]$, there is a vector $\left(\omega_{1}, \omega_{2}, \ldots, \omega_{n}\right)$ in $\left(\mathbf{F}_{q}[X][\rho]\right)^{n}$, which is periodic by the Dubois version of the Jacobi-Perron algorithm.

we prove also that there is no algebraic formal series $\omega$ such that the vector $\left(\omega, \omega^{2}\right)$ is 2-purely periodic by the homogenous version of Jacobi-Perron algorithm and we give a characterization of the vector $\left(\omega, \omega^{2}\right)$ which is 1 and 3 -purely periodic by it. We conjecture that this result holds for $2 n$ and $2 n+1$.

This paper is organized in the following way. In section 2, we present a brief review of the field of formal power series and we present the basic notions and notations needed throughout the work.. In section 3, we give the main results and propose a weaker conjecture which concerns purely periodic vector by $J$.

\section{The field of formal power series}

Let $\mathbb{F}_{q}$ be a field with $q$ elements of characteristic $p, \mathbb{F}_{q}[X]$ the set of polynomials of coefficients in $\mathbb{F}_{q}$ and $\mathbb{F}_{q}(X)$ its field of fractions. The set $\mathbb{F}_{q}\left(\left(X^{-1}\right)\right)$ of formal power series over $\mathbb{F}_{q}$ is defined as follows

$$
\mathbb{F}_{q}\left(\left(X^{-1}\right)\right)=\left\{\omega=\sum_{j=s}^{+\infty} a_{j} X^{-j}: a_{j} \in \mathbb{F}_{q}, a_{s} \neq 0 \text { with } s \in \mathbb{Z}\right\} .
$$

Let $\omega=\sum_{j=s}^{+\infty} a_{j} X^{-j} \in \mathbb{F}_{q}\left(\left(X^{-1}\right)\right)$, we denote its polynomial part by $[\omega]$ and $\{\omega\}$ its fractional part. We state that $\omega=[\omega]+\{\omega\}$.

A Pisot element $\omega \in F_{q}\left(\left(X^{-1}\right)\right)$ is an algebraic integer over $F_{q}[X]$ such that $|\omega|>1$ whose remainder conjugates in $\bar{F}_{q}\left(\left(X^{-1}\right)\right)$ have an absolute value strictly smaller than 1. For more information about Pisot element see $[13,6,9,10,11$, 12].

Recall that $F_{q}\left(\left(X^{-1}\right)\right)$ contains Pisot elements of any degree over $F_{q}(X)$. Indeed, consider the polynomial $Y^{d}-a X Y^{d-1}-1$, where $a \in F_{q} \backslash\{0\}$, it can be seen easily, considering its Newton polygon, that the polynomial, which is irreducible over $F_{q}(X)$, has a root $\omega \in F_{q}\left(\left(X^{-1}\right)\right)$ such that $|\omega|>1$ and all of its conjugates in $\bar{F}_{q}\left(\left(X^{-1}\right)\right)$ have an absolute value strictly smaller than 1 .

Notations: In all this paper, we denote by $J$ the Jacobi-Perron algorithm and by $D$ its Dubois version.

The Jacobi-Perron Algorithm: Let $M_{0}=\left\{\omega \in \mathbb{F}_{q}\left(\left(X^{-1}\right)\right)^{n}:|\omega|<1\right\}$ and $M=M_{0} \backslash\{0\} \times M_{0}^{n-1}$ the Jacobi-Perron algorithm is defined, for all $f=\left(f_{1}, \ldots, f_{n}\right) \in M$, by

$$
J(f)=\left(\frac{f_{2}}{f_{1}}-\left[\frac{f_{2}}{f_{1}}\right], \ldots, \frac{f_{n}}{f_{1}}-\left[\frac{f_{n}}{f_{1}}\right], \frac{1}{f_{1}}-\left[\frac{1}{f_{1}}\right]\right) \in M_{0}^{n} .
$$

We set $M^{*}=\left\{f \in M: J^{s}(f)\right.$ is well defined for all $\left.s \geq 1\right\}$. Let $f \in M^{*}$, we define $f^{(t)}=\left(f_{1}^{(t)}, \ldots, f_{n}^{(t)}\right)=J^{t}(f)$ and $k^{(t)}(f)=\left(k_{1}^{(t)}, \ldots, k_{n}^{(t)}\right)=\left(\left[\frac{f_{2}^{(t-1)}}{f_{1}^{(t-1)}}\right], \ldots,\left[\frac{1}{f_{1}^{(t-1)}}\right]\right)$, for all $t \geq 1$. We notice that $M^{\star}=\left\{f \in M:\right.$ for all $\left.s \geq 1, f_{1}^{(s)} \neq 0\right\}$. We claim that the sequence $\left(k^{(t)}\right)$ is unique. In fact, if $f \in M^{*}$, we have $\frac{1}{f_{1}^{(t)}}=\left[\frac{1}{f_{1}^{(t)}}\right]+\left\{\frac{1}{f_{1}^{(t)}}\right\}$, let $k_{n}^{(t+1)}=\left[\frac{1}{f_{1}^{(t)}}\right]$ and 
$f_{n}^{(t+1)}=\left\{\frac{1}{f_{1}^{(t)}}\right\}$. As $\mathbb{F}_{q}\left(X^{-1}\right)=\mathbb{F}_{q}[X] \oplus M_{0}$, the decomposition is unique. The relation $f_{i}^{(t+1)}=\frac{f_{i+1}^{(t)}}{f_{1}^{(t)}}-\left[\frac{f_{i+1}^{(t)}}{f_{1}^{(t)}}\right]$ defines in an unique way $f_{i}^{(t+1)}$ and $k_{i}^{(t+1)}$, for all $1 \leq i \leq n-1$, therefore $k_{n}^{(t+1)}$ has an unique value, so the sequence $\left(k^{(t)}\right)$ is unique.

The version of $J$ introduced by Dubois. Let $E=\left(\mathbb{F}_{q}\left(\left(X^{-1}\right)\right)\right)^{n}$. In order to have an algorithm defined in the whole space $E$ a version of (1) is introduced in [4]. For all $f=\left(f_{1}, \ldots, f_{n}\right) \in E, f_{1} \notin \mathbb{F}_{q}[X]$, we define the map

$$
D(f)=\left(\frac{f_{2}-\left[f_{2}\right]}{f_{1}-\left[f_{1}\right]}, \ldots, \frac{f_{n}-\left[f_{n}\right]}{f_{1}-\left[f_{1}\right]}, \frac{1}{f_{1}-\left[f_{1}\right]}\right) \in E .
$$

Let $f \in E^{\star}=\left\{f \in E: D^{s}(f)\right.$ is well defined for all $\left.s \geq 1\right\}$. For all $t \geq 1$, we set $f^{(t)}=D^{t}(f)=\left(f_{1}^{(t)}, \ldots, f_{n}^{(t)}\right)$ and

$$
a^{(t)}(f)=\left(a_{1}^{(t)}, \ldots, a_{n}^{(t)}\right)=\left(\left[f_{2}^{(t-1)}\right], \ldots,\left[f_{n}^{(t-1)}\right],\left[f_{1}^{(t-1)}\right]\right) .
$$

\section{Results}

Theorem 1. Any finite algebraic extension for $\mathbb{F}_{q}(x)$ in $\mathbb{F}_{q}\left(\left(X^{-1}\right)\right)$ can be generated by a Pisot element.

To prove this theorem, we need the following results ( see $[13,10])$.

Theorem 2. ( Theorem 1.2, $p$ 597, [13]) Let $\mathbb{K}$ be an arbitrary field. Then any finite separable extension of $\mathbb{K}(X)$ which is contained in $\mathbb{K}\left(\left(X^{-1}\right)\right)$ can be generated by a Pisot element.

Lemma 1. (Lemma 1.1, $p$ 599, [13]) If $\mathbb{K}$ is a perfect field, then any algebraic element of $\mathbb{K}\left(\left(X^{-1}\right)\right)$ is separable over $\mathbb{K}(X)$.

Lemma 2. Let $\mathbb{K}$ be an arbitrary field. If $\mathbb{K}$ is finite, then it is perfect i.e. all finite extension of $\mathbb{K}$ is separable.

Proof. Assume that there is an algebraic element of $\mathbb{K}$ whose minimal polynomial $P$ is in $\mathbb{F}_{q}\left[X^{p}\right]$, then we write

$$
P(X)=a_{0}+a_{p} X^{p}+a_{2 p} X^{2} p+\ldots \in \mathbb{F}_{q}\left[X^{p}\right] .
$$

If we set $Q(X)=\sigma^{-1}\left(a_{0}\right)+\sigma^{-1}\left(a_{p}\right) X+\sigma^{-1}\left(a_{2 p}\right) X^{2}+\ldots \in \mathbb{K}[X]$, where $\sigma$ is the automorphism defined over $\mathbb{K}$ by $\sigma: x \rightarrow x^{p}$, we obtain $P=Q^{p}$, which contradict the fact that $P$ is irreducible.

Proof. ( Theorem 1) As $\mathbb{F}_{q}$ is a finite field, then, by lemma 2, it is perfect. Using Lemma 1, any algebraic element of $\mathbb{F}\left(\left(X^{-1}\right)\right)$ is separable over $\mathbb{F}(X)$. Using Theorem 2 , the theorem is proved.

Now we are ready to prove one of our results.

Theorem 3. Let $b_{0}, a_{0}, \ldots, a_{n} \in \mathbb{F}_{q}[X]$ be such that $\left(a_{0}, b_{0}\right)=1,\left|a_{n}\right|>\left|a_{i}\right|$ and $a_{0}$ divides $a_{i+1}$, for all $0 \leq i \leq n-1$. Let $\rho$, where $|\rho|>1$, be a root of the polynomial

$$
b_{0} Y^{n+1}-b_{0} a_{n} Y^{n}-\ldots-b_{0} a_{1} Y-a_{0} .
$$

Let $f \in M^{\star}$ be the vector defined by

$$
\begin{aligned}
& f_{n}=\rho \\
& f_{n-1}=\rho^{2}-a_{n} \rho \\
& \vdots \\
& f_{1}=\rho^{n}-a_{n} \rho^{n-1}-\ldots-a_{2} \rho .
\end{aligned}
$$


(i) If $a_{0} \neq b_{0}$, then $f$ is periodic by $D$ with period $n+1$.

(ii) Let $h \in M^{\star}$, then $D(h)=h$ if, and only if, $h=f$ which is defined by a Pisot element for (4) with $a_{0}=b_{0}$. Moreover, the partial quotient vector $a(f)=\left(a_{1}, \ldots, a_{n}\right)$.

Proof. (i) Let $a_{0} \neq b_{0}$. By (4) and (5),

$$
f_{n}=\rho=a_{n}+\frac{a_{n-1}}{\rho}+\ldots+\frac{a_{1}}{\rho^{n-1}}+\frac{a_{0}}{b_{0} \rho^{n}} .
$$

As $|\rho|>1$ and $\left|a_{n}\right|>\left|a_{i}\right|$, for all $0 \leq i \leq n-1$, we have that $|\rho|=\left|a_{n}\right|$. Using again $|\rho|>1$ and $\left|a_{n}\right|>\left|a_{i}\right|$, for all $0 \leq i \leq n-1$, we conclude that $\left[f_{n}\right]=a_{n}$. Analogously, for all $1 \leq j \leq n-1$,

$$
f_{j}=\rho^{n+1-j}-a_{n} \rho^{n-j}-\ldots-a_{j+1} \rho=a_{j}+\frac{a_{j-1}}{\rho}+\ldots+\frac{a_{1}}{\rho^{j-1}}+\frac{a_{0}}{b_{0} \rho^{j}}
$$

which implies that $\left[f_{j}\right]=a_{j}$.

Let $D(f)=\left(f_{1}^{\prime}, \ldots, f_{n}^{\prime}\right)$, we have, for $1 \leq i \leq n-1$,

$$
f_{i}^{\prime}=\frac{f_{i+1}-\left[f_{i+1}\right]}{f_{1}-\left[f_{1}\right]}=\frac{f_{i+1}-a_{i+1}}{f_{1}-a_{1}} \text { and } f_{n}^{\prime}=\frac{1}{f_{1}-a_{1}} .
$$

On the other hand $f_{1}-a_{1}=\frac{a_{0}}{b_{0} \rho}=\frac{a_{0}}{b_{0} f_{n}}$ and $f_{i+1}-a_{i+1}=\frac{a_{i}}{\rho}+\ldots+\frac{a_{0}}{b_{0} \rho^{i+1}}=\frac{f_{i}}{\rho}$ which give

$$
f_{i}^{\prime}=f_{i} \frac{b_{0}}{a_{0}} \text { and } f_{n}^{\prime}=f_{n} \frac{b_{0}}{a_{0}}
$$

We have, for all $1 \leq i \leq n$, as $a_{0}$ divides $a_{i}$,

$$
\left[f_{i}^{\prime}\right]=\left[f_{i} \frac{b_{0}}{a_{0}}\right]=\left[\left(a_{i}+\frac{a_{i-1}}{\rho}+\ldots+\frac{a_{0}}{b_{0} \rho^{i}}\right) \frac{b_{0}}{a_{0}}\right]=\left[b_{0}\left(\frac{a_{i}}{a_{0}}+\frac{a_{i-1}}{a_{0} \rho}+\ldots+\frac{a_{0}}{a_{0} b_{0} \rho^{i}}\right)\right]=b_{0} \frac{a_{i}}{a_{0}}
$$

We assume that, for $1 \leq i \leq n-1$,

$$
D^{i+1}(f)=f^{(i+1)}=\left(f_{1} \frac{b_{0}}{a_{0}}, \ldots, f_{n-i-1} \frac{b_{0}}{a_{0}}, f_{n-i+1}, \ldots, f_{n}\right) .
$$

We have, for all $2 \leq j \leq n-i-1$,

$$
f_{j} \frac{b_{0}}{a_{0}}-\left[f_{j} \frac{b_{0}}{a_{0}}\right]=\frac{b_{0}}{a_{0}}\left(f_{j}-\left[f_{j}\right]\right)=\frac{b_{0} f_{j}}{a_{0} \rho} \text { and } f_{1} \frac{b_{0}}{a_{0}}-\left[f_{1} \frac{b_{0}}{a_{0}}\right]=\frac{b_{0}}{a_{0}}\left(f_{1}-\left[f_{1}\right]\right)=\frac{1}{\rho}
$$

and conclude that

$$
f_{j-1}^{(i+2)}=\frac{f_{j}^{(i+1)}-\left[f_{j}^{(i+1)}\right]}{f_{1}^{(i+1)}-\left[f_{1}^{(i+1)}\right]}=\frac{b_{0} f_{j}}{a_{0} \rho} \rho=\frac{b_{0}}{a_{0}} f_{j-1} .
$$

Besides, for $n-i \leq j \leq n$,

$$
f_{j-1}^{(i+2)}=\frac{f_{j}^{(i+1)}-\left[f_{j}^{(i+1)}\right]}{f_{1}^{(i+1)}-\left[f_{1}^{(i+1)}\right]}=\left(f_{j}-\left[f_{j}\right]\right) \rho=\frac{f_{j-1}}{\rho} \rho=f_{j-1} .
$$

We have obtained that, for $1 \leq i \leq n-2$,

$$
D^{i+2}(f)=\left(f_{1} \frac{b_{0}}{a_{0}}, \ldots, f_{n-i-1} \frac{b_{0}}{a_{0}}, f_{n-i}, \ldots, f_{n}\right)
$$


which implies that $D^{n+1}(f)=f$ and $D^{k}(f) \neq f$, for all $1 \leq k \leq n$.

(ii) Let $a_{0}=b_{0}$ and $f$ be given by (5), then $D(f)=f$ by (6). Conversely assume $D(h)=h$, where $h \in M^{\star}$. Let $b_{j}=\left[h_{j}\right]$, for all $1 \leq j \leq n$, thus

$$
\left|b_{n}\right|>\left|b_{i}\right| \text {, for all } 1 \leq i \leq n-1 .
$$

Let $f$ be defined by (5.5) using $a_{1}=b_{1}, \ldots, a_{n}=b_{n}$ and $a_{0}=b_{0}=1$. Therefore $D(f)=f$, and moreover the sequence $H_{0}^{(t)}, H_{1}^{(t)}, \ldots, H_{n}^{(t)}$ is the same for $h$ and $f$. As Dubois algorithm converges [4], the uniqueness of the limit gives $h=f$.

According to ( Theorem 1), the above theorem assure the following result.

Theorem 4. Over any formal power series field extension of degree $(n+1), \mathbb{F}_{q}[X][\rho]$, there is a vector $\left(\omega_{1}, \omega_{2}, \ldots, \omega_{n}\right)$ in $\left(\mathbb{F}_{q}[X][\rho]\right)^{n}$, which is periodic by the Dubois version of the Jacobi-Perron algorithm.

In the real case, Bouhamza [7] characterized the vector of form $\left(\alpha, \alpha^{2}\right)$ which is 1-periodic by Jacobi-perron algorithm of pre-period 2. Note that he uses a different form of Jacobi-Perron algorithm (see [7]). He obtained also the following results.

Theorem 5. There is no algebraic real $\alpha$, of degree 3, such that the vector $\left(\alpha, \alpha^{2}\right)$ is 2-purely periodic by $J$.

Theorem 6.There is no real $\alpha$ such that the vector $\left(\alpha, \alpha^{2}\right)$ is 1- purely periodic by $J$.

Remark. Theorem 6 is correct if $\alpha>1$. But if $0<\alpha<1$ then one uses a slightly different form which is given in (1).

We give the analogous of these results in the case of formal power series.

Theorem 7. Let $\omega \in M^{*}$, the vector $\left(\omega, \omega^{2}\right)$ is purely 1-periodic by $J$ if and only if $\omega$ is the inverse of a Pisot cubic element $v$ such that $v^{3}-A v-1=0$, where $A \in \mathbb{F}_{q}[X] \backslash F_{q}$.

Theorem 8. There is no vector $\left(\omega, \omega^{2}\right)$ which is purely 2-periodic by $J$.

Remark. The proof of theorem 7 and theorem 8 is left as an exercise for the interested reader.

Theorem 9. Let $\omega \in M^{*}$. The vector $\left(\omega, \omega^{2}\right)$ is 3-purely periodic by $J$ if and only if $w$ is the inverse of a Pisot cubic element $v$ such that $A v^{3}+A B v-B=0$, where $A, B \in \mathbb{F}_{q}[X] \backslash F_{q}$.

Proof. Let $\omega \in M^{*}$, the vector $\left(\omega, \omega^{2}\right)$ is purely 3-periodic by $J$, then

$$
\begin{gathered}
J\left(\omega, \omega^{2}\right)=\left(\omega, \frac{1}{\omega}-\left[\frac{1}{\omega}\right]\right) . \\
J^{2}\left(\omega, \omega^{2}\right)=\left(\left\{\frac{\left\{\frac{1}{\omega}\right\}}{w}\right\},\left\{\frac{1}{\omega}\right\}\right) .
\end{gathered}
$$

and

$$
\begin{aligned}
J^{3}\left(\omega, \omega^{2}\right) & =\left(\frac{\left\{\frac{1}{\omega}\right\}}{\left\{\frac{\left\{\frac{1}{\omega}\right\}}{w}\right\}},\left\{\frac{\omega}{\left\{\frac{1}{\omega}\right\}}\right\}\right) . \\
& =\left(\omega, \omega^{2}\right)
\end{aligned}
$$


then,

$$
\omega^{2}=\left\{\frac{\omega}{\left\{\frac{1}{\omega}\right\}}\right\}=\frac{\omega}{\frac{1}{\omega}-\left[\frac{1}{\omega}\right]}-\left[\frac{\omega}{\frac{1}{\omega}-\left[\frac{1}{\omega}\right]}\right]
$$

which gives

$$
\left[\frac{1}{\omega}\right] \omega^{3}+\left[\frac{\omega}{\frac{1}{\omega}-\left[\frac{1}{\omega}\right]}\right]\left[\frac{1}{\omega}\right] \omega-\left[\frac{\omega}{\frac{1}{\omega}-\left[\frac{1}{\omega}\right]}\right]=0
$$

Let $v=\frac{1}{\omega}$, we set $A=\left[\frac{\omega}{\frac{1}{\omega}-\left[\frac{1}{\omega}\right]}\right]$ and $B=\left[\frac{1}{\omega}\right]$, thus, we have

$$
A v^{3}-A B v^{2}-B=0
$$

it is clear that $A, B \in F_{q}[X] \backslash F_{q}$. Now, assume that $v$ is the root of the polynomial $P(Y)=A Y^{3}-A B Y^{2}-B$ with $A, B \in$ $F_{q}[X] \backslash F_{q}$, such that $|v|>1$, it is clear that $[v]=B$ and we have $A v^{2}(v-B)=B$ so,

$$
\begin{aligned}
A & =\frac{[v]}{v^{2}\{v\}} \\
& =\frac{1}{v\{v\}} \frac{[v]}{[v]+\{v\}} \\
& =\frac{1}{v\{v\}} \frac{1}{1+\frac{\{v\}}{[v]}} \\
& =\frac{1}{v\{v\}}\left(1-\frac{\{v\}}{[v]}+\frac{\{v\}^{2}}{[v]^{2}}-\cdots\right) \\
& =\frac{1}{v\{v\}}-\frac{1}{v[v]}+\frac{\{v\}}{v[v]^{2}}-\cdots
\end{aligned}
$$

which implies that $A=\left[\frac{1}{v[v]}\right]$.

$$
\begin{gathered}
J\left(\frac{1}{v}, \frac{1}{v^{2}}\right)=\left(\left\{\frac{1}{v}\right\},\{v\}\right) . \\
J^{2}\left(\frac{1}{v}, \frac{1}{v^{2}}\right)=(v\{v\},\{v\}) . \\
J^{3}\left(\frac{1}{v}, \frac{1}{v^{2}}\right)=\left(\left\{\frac{1}{v}\right\},\left\{\frac{1}{v\{v\}}\right\}\right)=\left(\frac{1}{v}, \frac{1}{v^{2}}\right) .
\end{gathered}
$$

This proves the Theorem.

It is natural to conjecture that the same claim holds for vectors $\left(\omega, \omega^{2}\right)$ which is $(2 n)$-purely periodic by $J$.

Remark. Take $\left(\omega, \omega^{2}\right)$ where $\omega$ is the root of $\omega^{3}+X^{2} \omega-X=0$, then it has a 3- periodic expansion. The difference seems to be that $A$ and $B$ can have common divisor.

Conjecture 2 . There is no vector $\left(\omega, \omega^{2}\right)$ which is $(2 n)$-purely periodic by $J$.

We assume characterize the vector $\left(\omega, \omega^{2}\right)$ which is purely $(2 n+1)$-purely periodic by $J$. 
Remark. Note that for $n=2$, the conjecture (2) is wrong for the real Jacobi-Perron algorithm : take $\omega$ as the root of $2 \omega^{3}+\omega^{2}+2 \omega=1$ which gives a 4 -periodic expansion.

\section{An application}

Let $\omega=\left[X, X^{2}, \cdots, X^{2^{s}}, \cdots\right]=X+\frac{1}{X^{2}+\frac{1}{\cdots+\frac{1}{X^{2^{s}}+\cdots}}} \in \mathbb{F}_{2}\left(\left(X^{-1}\right)\right)$ be a root of the cubic polynomial

$$
P(Y)=Y^{3}-X Y^{2}-1
$$

We will show that the expansion of the vector $\omega^{(0)}=\left(\frac{1}{\omega}, \frac{1}{\omega^{2}}\right)$ by $J$ is purely 1-periodic. We remark that $(\omega-X) \omega^{2}=1$ which implies that

$$
\omega=X+\frac{1}{\omega^{2}}
$$

thus $[\omega]=X$. We can apply $J$ at $\omega^{(0)}$ and denote $\omega^{(t)}=J^{t}\left(\omega^{(0)}\right)$. We have $\omega^{(1)}=\left(\frac{1}{\omega}, \omega-X\right)=\left(\frac{1}{\omega}, \frac{1}{\omega^{2}}\right)$. So, $\omega^{(1)}=$ $\omega^{(0)}$. Hence, the expansion is purely 1 -periodic.

\section{Acknowlegments}

All our thanks to the referee for careful reading of this manuscript, his corrections and many important remarks. We would like to thank Mohamed Mkaouar for suggesting to us the problem and for helpful discussions. We thank also Sa $\ddot{a} \mathrm{~d}$ Chandoul and Massöuda Loörayed for helpful discussions and many remarks.

\section{Competing interests}

The authors declare that they have no competing interests.

\section{Authors' contributions}

All authors have contributed to all parts of the article. All authors read and approved the final manuscript.

\section{References}

[1] A. Chandoul, M. Jellali, M. Mkaouar, Irreducible polynomials and number of Pisot elements (preprint).

[2] E. Dubois and R. Paysant-Le Roux, Algorithme de Jacobi-Perron dans les extensions cubiques. C. R. Acad. Sc. Paris, t. 280 (1975).

[3] E. Dubois and R. Paysant-Le Roux, Une application des nombres de Pisot à l'algorithme de Jacobi-Perron, Mh. Math, 98, (1984) 145-155

[4] E. Dubois, Algorithme de Jacobi-Perron dans un corps de séries formelles. Séminaire Delange-Pisot-Poitou. Théorie des nombres, tome 13, n-1 exp, n-8,(1971-1972) 1-11.

[5] H. Ben amar, A. Chandoul, Convergence of the Brun algorithm over the field of formal power series, J. Number Theory 129 (2009) 621-631.

[6] M.J. Bertin, A. Decomps-Guilloux, M. Grandet-Hugot, M Pathiaux-Deleosse, J.P, Schreiber, Pisot and Salem numbers. (1992) Birkhauser Verlag Basel. 
[7] M. Bouhamza, Algorithme de Jacobi-Perron dans le corps de nombres de degré 3, Bull. Sc. math, $2^{e}$ série, 108 ( 1984) $101-111$.

[8] M. Bouhamza, Algorithme de Jacobi-Perron dans le corps de nombres de degré 4, Acta Arithm. 44, 141-145.

[9] M. Grandet-Hugot, Sur une propriété des nombres de Pisot dans un corps de série formelles, C.R.A.S., 266, ser. A, (1967), A39-A41.

[10] M. Grandet-Hugot, Nombre de Pisot dans un corps de série formelle, Séminaire Delange-Pisot-Poitou. Théorie des nombres, tome 8, n1 (1966-1967) exp. n4, p 1-12., 14 (1968) 177-184.

[11] M. Grandet-Hugot, Eléments algébriques remarquables dans un corps de série formelles, Acta Arith., 14 (1968) 177-184.

[12] M. Hbaid and M. Mkaouar, Sur le béta développemnt de 1 dans le corps des séries formelles, Int. J. Number Theory 2 (3) (2006) 365-378.

[13] P. Bateman and A. L. Duquette, The analogue of Pisot-Vijayaraghavan numbers in fields of power series, Ill. J. Math., 6 (1962) 594-406.

[14] Paley, Ursell, Continued fraction in several dimensions, Proc. Cambride Phil. Sco. 26( 1930) 127-144.

[15] Perron, Ein Stazüber Jacobi-Ketten zweiter Ordung, Ann. R. Sc. Norm. Super Pisa, fis. mat. (2) 4 (1935) 133-138. 